\title{
MCPyV TAg-specific Polyclonal Autologous CD8-positive T Cells
}

National Cancer Institute

\section{Source}

National Cancer Institute. MCPyV TAg-specific Polyclonal Autologous CD8-positive T

Cells. NCI Thesaurus. Code C104009.

A preparation of polyclonal autolog ous CD8 positive T-lymphocytes specific for the Merkel cell polyomavirus (MCPyV) T antigen ( $\mathrm{TAg}$ ) with potential antineoplastic activity. Peripheral blood lymphocytes from a Merkel cell carcinoma (MCC) patient were obtained and antigen-specific CD8+ T cells targeting a specific MCPyV TAg epitope were derived and expanded ex vivo. Upon infusion of the MCPyV TAg-specific polyclonal autologous CD8-positive T cell vaccine, the T cells recognize the MCPyV antigen and exert a cytotoxic T-lymphocyte response against the MCPyV TAg-expressing MCC cells. MCPyV is expressed in about $80 \%$ of MCC and is not expressed in normal, human tissue; the MCPyVT ag oncoprotein plays a key role in MCC survival and tumor cell proliferation. 